\title{
MEASURING TECHNOLOGICAL, ORGANIZATIONAL AND ENVIRONMENTAL FACTORS INFLUENCING THE ADOPTION INTENTIONS OF PUBLIC CLOUD COMPUTING USING A PROPOSED INTEGRATED MODEL
}

\author{
Dr. Minimol Anil Job \\ Assistant Professor, ITC Department, Faculty of Computer Studies, \\ Arab Open University, Kingdom of Bahrain
}

\begin{abstract}
The main objective of this research is to identify the factors influencing the intentions to adopt the public computing by the private sector firms. In this research the researcher examined the ten factors influencing the cloud computing adoption using a proposed integrated model which incorporates aspects of the Technology, Organization and Environment factors such as Complexity, Compatibility, Security Concerns, Trialability, Cost Saving, Top Management Support, Prior IT Experience, Organizational Readiness, Competitive Pressure and External Support. In order to test influencing factors a survey was conducted and one hundred and twenty two valid responses were received from IT decision makers from forty firms in different industries. The results revealed that the Compatibility, Cost Saving, Trialability and External Support are the main influential factors in the adoption intentions of public cloud computing. Future research could be built on this study by developing different model for each industry because each industry has unique characteristics that can influence the adoption of the technological innovations.
\end{abstract}

\section{KEYWORDS}

cloud computing, virtualization, security, Compatibility, Complexity, Trialability

\section{INTRODUCTION}

Due to the intense market competition and a rapidly changing business environment, firms have been driven to adopt various modern information technologies in order to improve their business operations and increasing their productivity [1]. Since the private sector firms are important players in each industry which significantly contribute to the economy's Gross domestic product and labor force, it is important to propose new strategies and technologies that can help 
the private sector firms to become more efficient and effective. The high cost of computing technologies is due to complex information architecture and infrastructure, and that will discourage the firms from adopting advanced IT services [2]. Based on that, one approach that helps the firms to enhance the productivity and being efficient is to invest in public cloud computing. . Cloud computing offers several benefits for enterprises. The cloud frees organizations from having to set up an IT infrastructure and allows them to rent resources and pay only for the services they use [3]. Yet, the emergence of cloud computing solves this problem by reducing direct expenses of information technology. For many firms, the adoption of public cloud computing became more beneficial as it can quickly add more capabilities to their IT systems without investing in new expensive infrastructure, buying or deploying new application systems, or training new IT personnel. The concerns related to the clients' data privacy and protection, problems with data separation in the cloud and long-term viability of the public cloud provider can negatively affect the firms' willingness to adopt the public cloud computing. Thus such firms are mostly hesitant to adopt the public cloud services. This research studied the factors that influence the adoption intentions of public cloud computing by the IT decision makers in the private sector firms using a proposed integrated model and the research aims to spot the light to the adoption intentions of cloud computing in a wide range of private sector firms from different industries, as well as contributing to the body of knowledge related to the factors of adoption.

\section{METHODOLOGY}

Public Cloud Computing is one of the emerging areas in the field of information technology. Cloud computing is considered in the second place after business intelligent on the list of the top five most influential technologies. However, despite the fact that the adoption of public cloud computing has been growing, its rate of acceptance remains practically very low in the developing countries to about $37 \%$, and the rate expected to be even lower in the less developed countries as the case with most of the technological innovations [1]. Therefore, it is important to investigate the factors that affect the adoption intentions of public cloud computing by IT decision makers in the private sector firms. [4][5]The diagram below is a model proposed model to identify the factors influencing the public cloud adoption in the private sectors. The ten factors influence in the cloud computing adoption is examined in this research is using the proposed integrated model incorporates aspects of the Technology, Organization and Environment factors such as Complexity, Compatibility, Security Concerns, Trialability , Cost Saving, Top Management Support, Prior IT Experience, Organizational Readiness, Competitive Pressure and External Support [6][7].

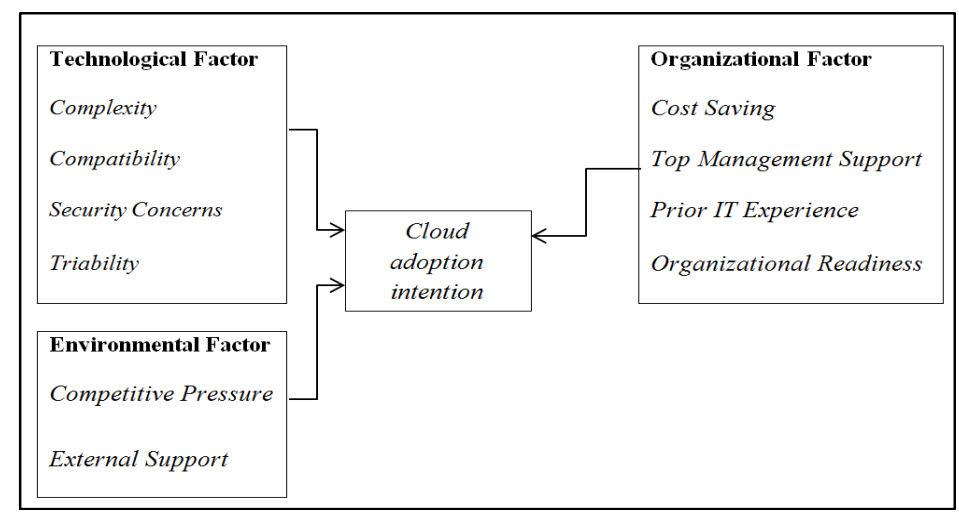

Figure1: A proposed model for cloud computing adoption 
The main question of this research is "What are the factors that influence the intentions in adopting public cloud computing in the private sector firms?" with the following main objectives.

- Analyze the technological, organizational and environmental factors influencing the IT decision maker intentions to adopt public cloud computing in the private sector.

- Provide suggestions and recommendations for the firms and the service providers in order to increase the adoption rate of public cloud computing between the firms in the private sector.

The targeted population in this research is defined as "managers and professionals who are involved in the decision process for the adoption of a new information technology in large firms in the private sector"[10][11]. This measure taken in this research to avoid small and medium firms based on the assumption that the large firms have more knowledge about the cloud computing because they mostly have experience with its related technologies such as virtualization and utility computing. As part of this research the researcher measured the degree of satisfaction in the firms already adopted the cloud services. A list of 30 firms preferably known as leader firms in different industries have selected as the target population[8][9]. The senior personnel of the IT department in these firms were contacted to in identifying the relevant hypothetical respondents for this survey within their firms as the targeted sample. An online questionnaire survey was conducted among the participants.

\section{DATA ANALYSIS}

\section{Respondents' Profile:}

All the participants in this research are IT decision makers who are familiar with public cloud computing. In addition to the participants' job titles, the type of industry in which the firms conducted their business was also captured. In terms of the respondents' profile, the collected data indicated that $76 \%$ of the respondents were not adopting public cloud computing services in their firms, while $29 \%$ were already adopting. Figure 3 views the respondents adoption status of public cloud computing.

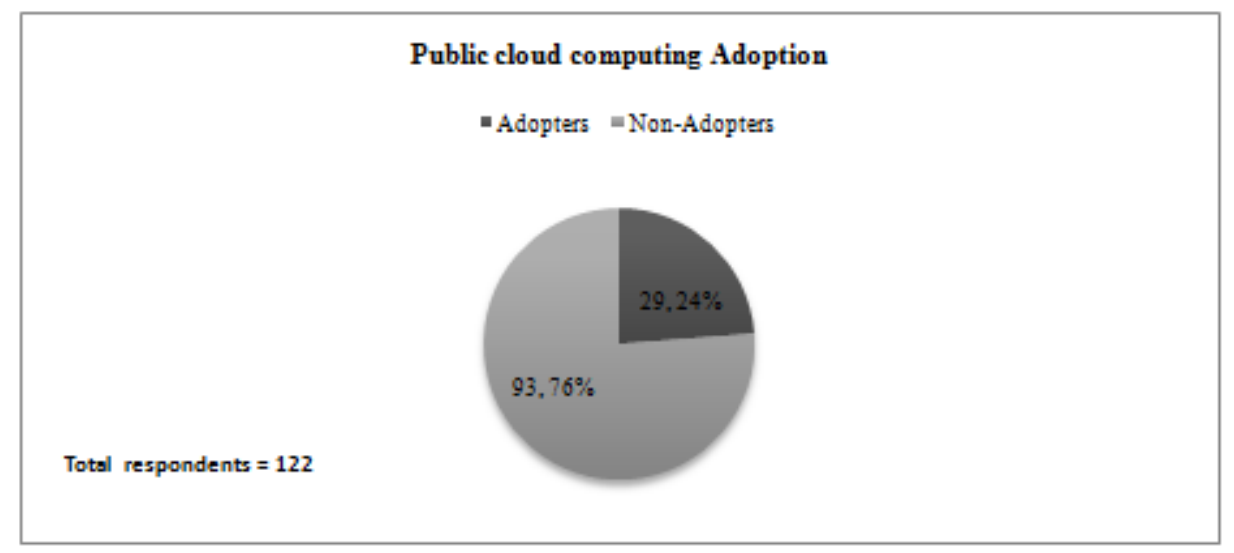

Figure 2: respondents' adoption status of public cloud computing 


\section{$\underline{\text { Technological Factors }}$}

\section{Complexity:}

As shown in Figure 3, only $27 \%$ of the respondents agreed with the fact that the work with cloud computing is complicated and about $26 \%$ agreed that the integration between their current IT infrastructure and cloud based services is difficult. At last, about $19 \%$ of the respondents agreed with the fact that administrating and monitoring the cloud based services are complex tasks.

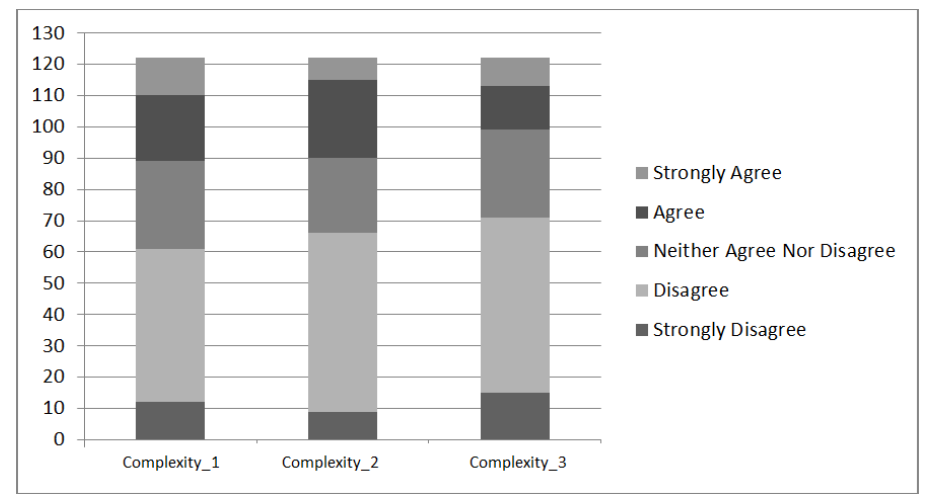

Figure 3: Analysis of respondents' perception about Complexity

\section{Compatibility:}

Using three questions, this construct measures the degree to which cloud computing is perceived as consistent with the existing infrastructure, culture and previous practices of the firms. As it can be viewed in Figure 4, about $66 \%$ of respondents think that cloud computing is compatible with the business model of their firms (Compatibility_1). More than 60\% agreed that the adoption of cloud computing is compatible with the norms and culture of their firms (Compatibility_2). About $61 \%$ think that cloud computing is compatible with their current IT infrastructure (Compatibility_3).

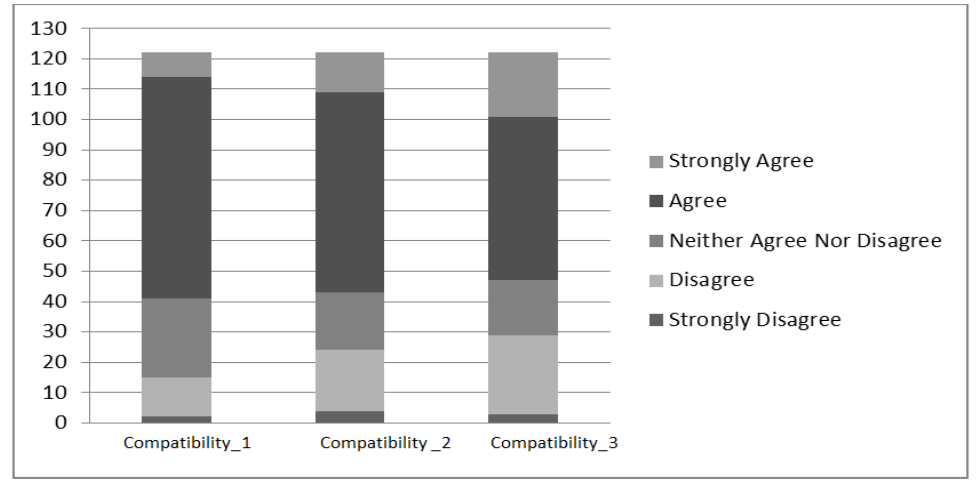

Figure 4: Analysis of respondents' perception about Compatibility

\section{Security Concerns:}

As shown in Figure 5, 46\% of respondents think that it is unsecured to keep their business data in the Cloud providers' data center (Security_1). About $41 \%$ claim that it is unsecured to use the 
cloud services over the internet to conduct their business' operations (Security_2), and 38.5\% claim that the cloud computing concept does not satisfy their firms' security and privacy policies (Security_3).

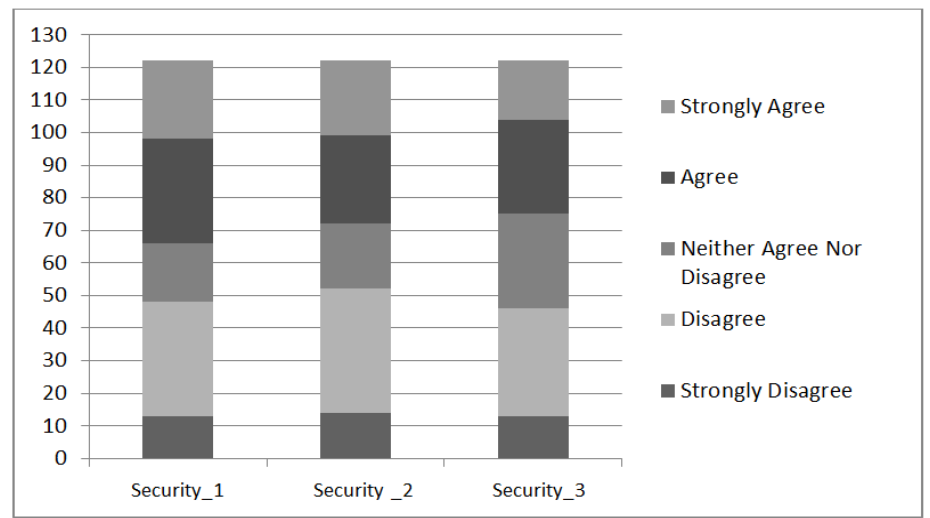

Figure 5: Analysis of respondents' perception about the security concerns

\section{Trialability:}

As shown in Figure 6, more than $70 \%$ of the participants think that before taking the adoption decision they will have the opportunity to use cloud computing services on a trial basis (Triability_1) and run partial integration test between the cloud applications and their existing system (Triability_2). Also, about 58\% agreed that the cloud providers offer their services on a trial basis long enough to prove the platform capabilities and benefits (Triability_3).

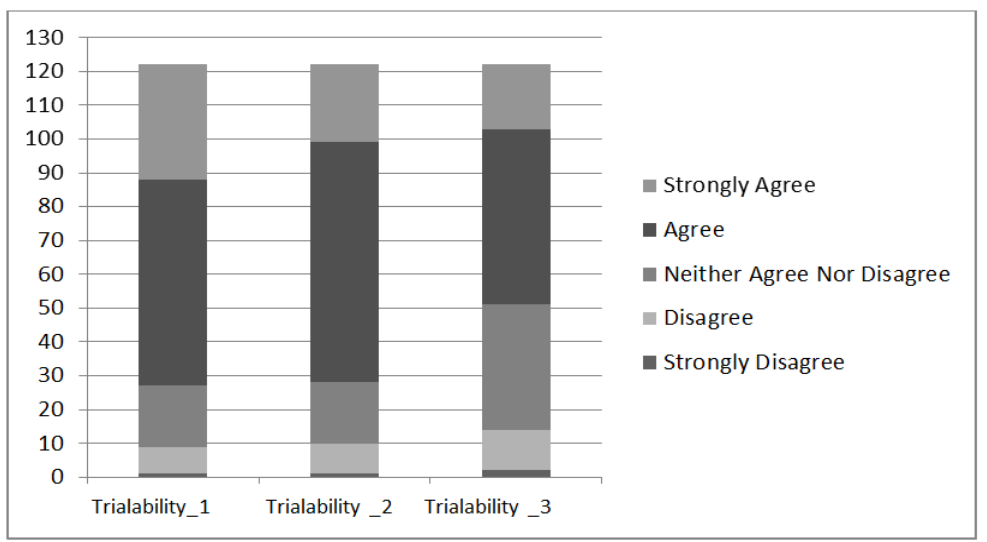

Figure 6: Analysis of respondents' perception about Perceived Trialability

\section{$\underline{\text { Environmental Factors }}$}

\section{Competitive Pressure:}

Figure 7 depicted that more than $75 \%$ of the respondents perceive very intense competition within their industry. About $72 \%$ claimed that their competitors are always looking for the technological innovation to gain competitive advantages, and about $63 \%$ of respondents believe that cloud computing can enhance the competitive power of their firms. 


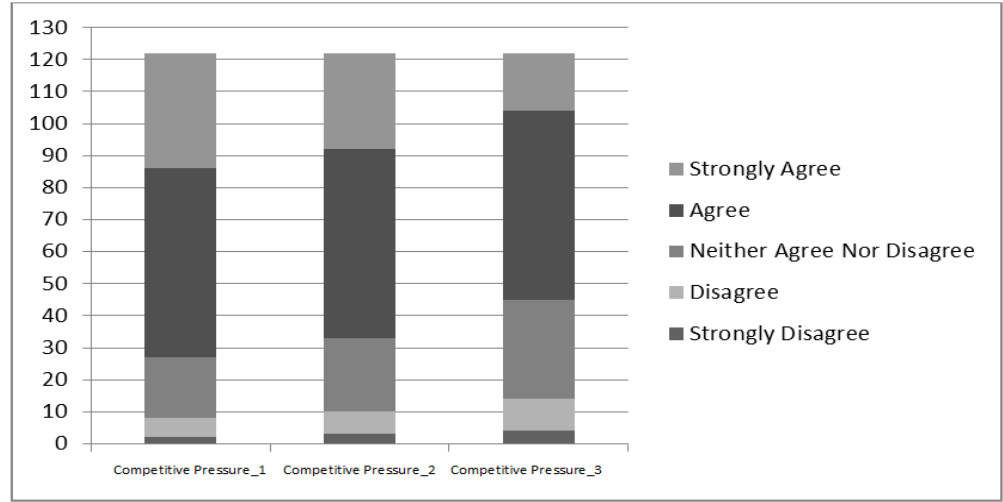

Figure 7: Respondents' perception about Competitive Pressure

\section{External Support:}

External support measured the participants expectations about the training program (External_Support_1); the technical support (External_Support_2); and the live support (hotline) provided by major cloud providers in the market (External_Support_3). Figure 8 shows the overall responses to each one. More than $80 \%$ of respondents believe that the level of external support delivered by cloud providers in each question either good, very good or excellent.

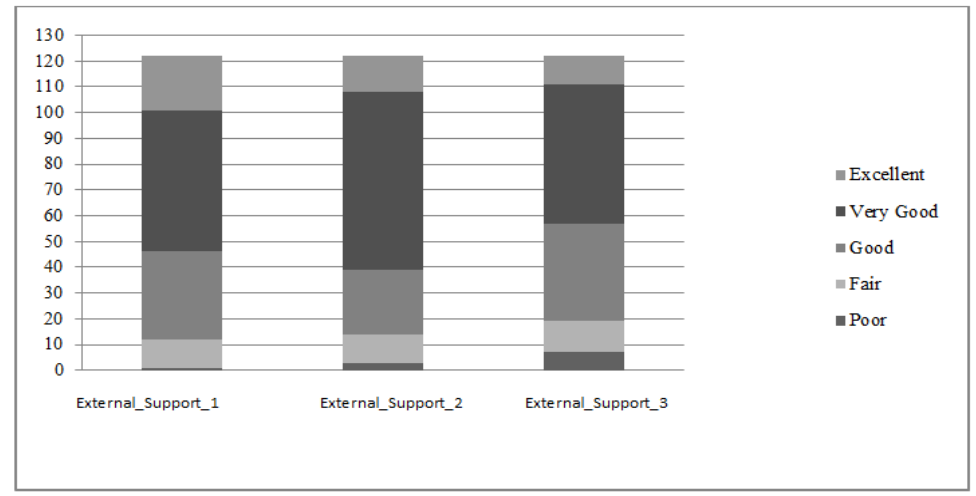

Figure 8: Analysis of respondents' perception about External Support

\section{$\underline{\text { Organizational Factors }}$}

\section{Cost Saving:}

The participants opinion about the cost of adopting public cloud computing is measured from different perspectives. As it can be observed from Figure 9, about $77 \%$ think that the benefits of cloud computing are greater than the costs of its adoption (Cost_Saving_1). Around 73\% agreed that acquiring applications systems through cloud computing is more economical than developing it in-house (Cost_Saving_2), and 62\% of the participants think that adopting an application system via cloud computing is more economical than purchasing it as Off-the-Shelf (Cost_Saving_3). 


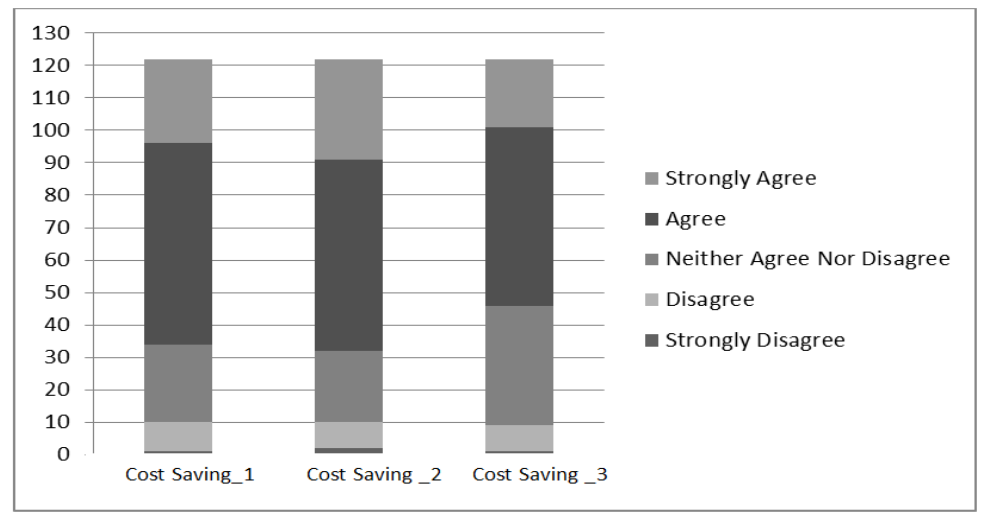

Figure 9: Analysis of respondents' perception about Cost Saving

\section{Top Management Support:}

Top management support is defined as the degree to which top management in the firm provides adequate resources and encourages the adoption of new technological innovations. As it can be viewed in Figure 10, about 70\% agreed that their top management supports the implementation of the new technological innovations (Top_Mgt_Support_1) and provides strong leadership and engagements when it comes to the adoption of new technology (Top_Mgt_Support_2). At the same time, only $52 \%$ think that their top management is willing to take the responsibility of unfavorable consequences related to the adoption of the new technology (Top_Mgt_Support_3).

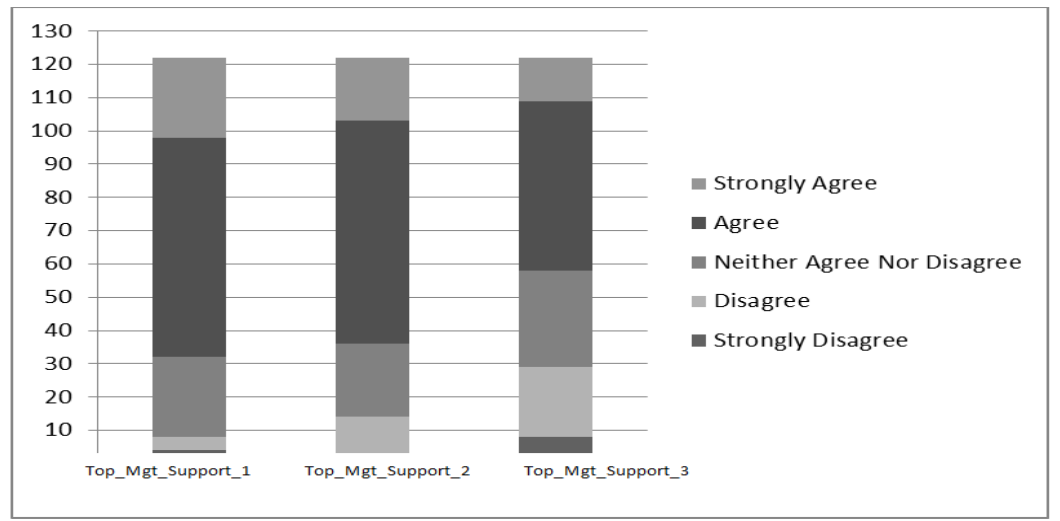

Figure 10: Analysis of respondents' perception about Perceived Top Management Support

\section{Prior IT Experience:}

Three questions were used to measures the firms' previous experience in the virtualization (Prior_IT Experience_1); Cluster computing (Prior_IT Experience_2); and Multi-Tenancy software architecture (Prior_IT Experience_3). Figure 11 shows that, 79\% of the participants have enough experience in virtualization technology in their firms, $81 \%$ claimed that they have experience in cluster computing, and about $73 \%$ have experience in multi-tenancy software architecture. 


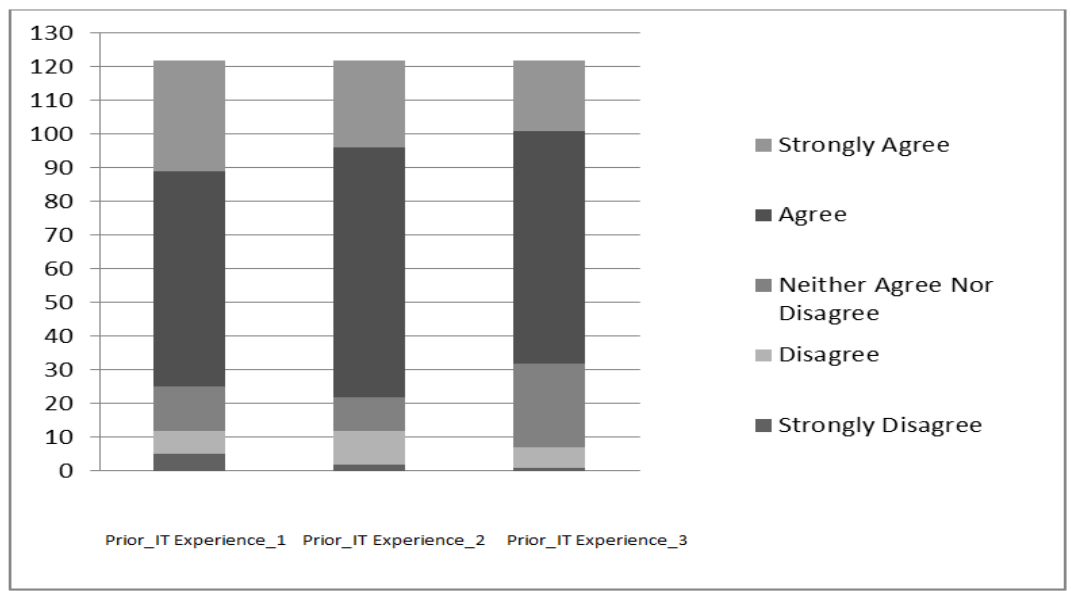

Figure 11: Analysis of respondents' perception about Prior IT experience

\section{Organizational Readiness:}

In this research the organizational readiness is defined as the degree to which the organization is technically and financially prepared to implement the cloud computing. As viewed in Figure 12, $72 \%$ of the participants claimed that their firms have sufficient financial resources to adopt cloud computing (Organizational_Readiness_1); 77\% think that their firms have the technological resources to adopt cloud computing (Organizational_Readiness_2); and about 75\% claimed that they have a qualified IT staff in order to adopt cloud computing (Organizational_Readiness _3).

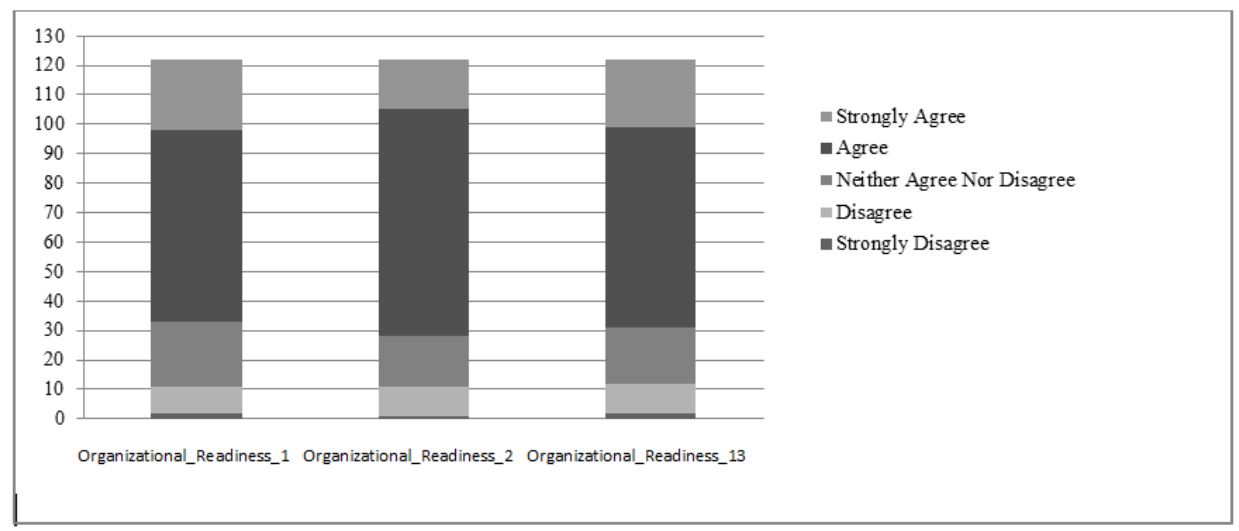

Figure 12: Analysis of respondents' perception about Organizational Readiness

\section{Satisfaction Level:}

For the participants who are already adopted the public cloud computing in their firms, we asked whether they are satisfied with the services received from cloud provider. As it can be seen in Figure $13,79 \%(24 \%+55 \%)$ of participants are satisfied with the service they receive. 


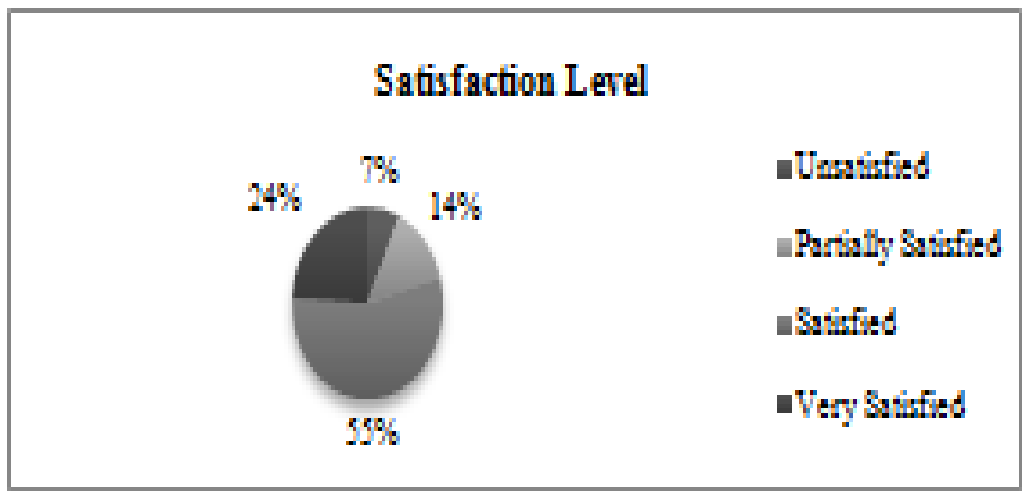

Figure 13: Cloud Adopters' Satisfaction

\section{Adoption Intentions:}

Only respondents, who have not adopted cloud computing yet, answered the questions related to the adoption intentions. The respondents asked whether the concept of cloud computing is acceptable in their firms (Adoption_Int_1); whether they recommend to use the cloud based applications in their firms in the future (Adoption_Int_2); whether they intent to adopt cloud computing in the coming two years (Adoption_Int_3); and whether they plan to adopt cloud computing in the next coming two years (Adoption_Int_4). As Figure 14 shows, about $72 \%$ of the non-adopters think that the concept of public cloud computing is acceptable in their firms and recommended the adoption of cloud based applications. Also, about 74\% think that they should adopt (or plan to adopt) public cloud based applications in coming two years. Overall the intention to adopt public cloud computing is high.

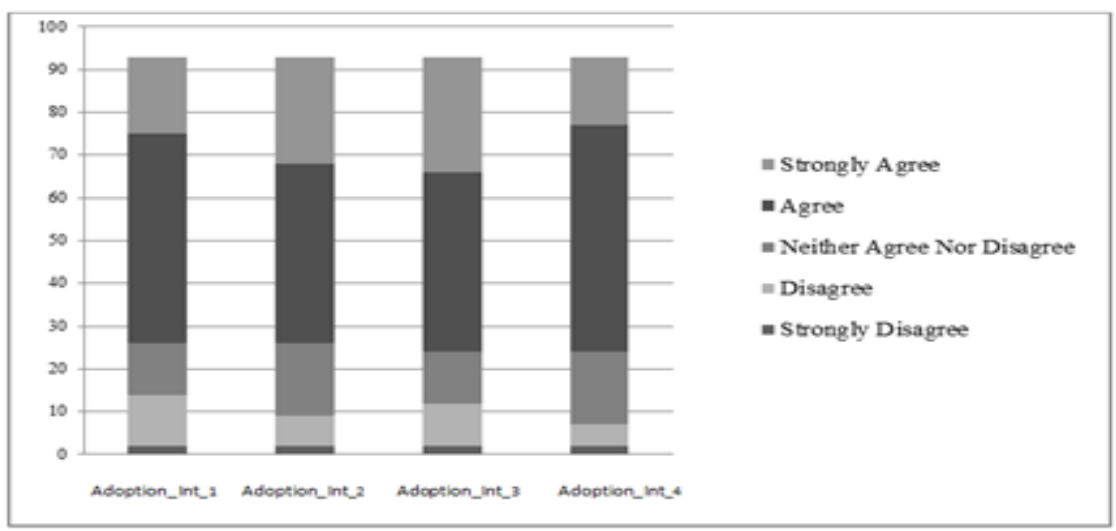

Figure 14: Analysis of non-adopters Intentions to adopt cloud computing

\section{CONCLUSiON}

This research tries to increase the theoretical background about public cloud computing adoption in the private sector firms in the developing countries from different points of views by adopting a complete framework that incorporates Technological, Organizational and Environmental factors to examine the intentions adoption of cloud computing. This research demonstrates several key 
findings about the factors influencing the adoption intentions of public cloud computing in private sector firms. These key findings are as follows:

- Four variables (Compatibility, Cost Saving, Trialability, and External support) were found to be significant determinants of public cloud computing adoption.

- Two variables (Organizational readiness, prior IT experience) were found to be positively related to the intentions to adopt the public cloud computing. However they have no significant impact.

- Three variables (Complexity, Security concerns and Competitive presser) were found to be negatively related to the intentions to adopt the public cloud computing but they have no significant impact.

- Among the determinants, Cost saving and External support were observed to be the most influential factor affecting the firm's adoption intentions.

According to the findings, there was no agreement between the participants on whether to consider the public cloud computing as secure or not. This resulted from the fact that the cloud computing concept still needs more time in order to prove its security capabilities and benefits to firms. Accordingly, when the firm is willing to adopt public cloud computing, it is better to start with services that do not mandates storing the critical business data in the provider's storage such as Virtual PBX phone service, Desktop as a Service, and Microsoft Office 365. [13][14] This will help the firm to be more knowledgeable about the capabilities exist within the cloud computing before implementing critical services such as ERP and CRM.

In order to be prepared for cloud computing, it is essential for the firm to optimize its network by installing intelligent load balancers in their infrastructure. Those devices allow the firm to manage, redirect and priorities the network traffic belong to mission-critical cloud applications when their bandwidth is under pressure, as well as allowing to scale up capacity over peak times. Also, it is important to analyze the existing IT assets to identify the tools that can re-use in the cloud without any modification and estimate how much effort (in terms of new development and deployment effort) will be required to integrate them with cloud based services before taking the adoption decision. [15][16] On the other hand, the cloud providers need to be aware of the concerns that firms experience when they make their adoption decision, such as cost saving, trialability and external support. The cloud providers should utilize various mass media in order to convince the firms that the migration to cloud-based system can help to dramatically reduce not only the fixed costs (such as licensing, hardware, software, storage, etc.), but also operating costs (such as cost of infrastructure administration, hardware and software maintenance, systems updates and upgrade, etc.) as well as training costs[17]. In addition, the service provider must arrange their services in order to be experimental. Giving the opportunity for the firms to try the services in experimental environment long enough to prove the platform capabilities and concept which more likely result an increase in the adoption rate. Although the of the majority participants in this research perceive the cloud services as more likely to be trialable ,it is necessary for service providers to make trialability more convenient and accessible. Furthermore, the cloud provides should support their clients according to a comprehensive service level agreement that assure high quality remote and onsite technical support[18]. That will allow the firms to obtain assistance for suspected defects and get valuable answers for task-oriented 
questions related to the installation and operation of the currently acquired services. In addition, it is essential to maintain a 24/7 responsive support service, and to offer electronic access to the provider's technical support knowledgebase and technical product specialists.

\section{RECOMMENDATIONS}

Future research could be built on this study by developing different model for each industry other than a comprehensive one, because each industry has unique characteristics that can influence the adoption of the technological innovations. In addition, this research investigates only the large firms which in turn limit the ability to generalize the findings. Therefore, the future researches could investigate the small and medium-sized enterprises (SMEs) in addition to the large ones. In order to enhance the data quality in future researches, it is essential to conduct semi-structured interview in addition to the survey. This approach is useful in delving into business-related decision analysis and gives the opportunity for the researchers to interact directly with the decision-makers to clarify any misleading points related to the data collection instruments.

\section{REFERENCES}

[1] Empirical Analysis for the Factors Affecting the Adoption of Cloud Computing Initiatives by Information Technology Executives Journal of Management Research ISSN 1941-899X 2013, Vol. 5, No. 1

[2] A Complete History of Cloud Computing . (2012, January). Retrieved October 5, 2013, from SalesForce: http://www.salesforce.com/uk/socialsuccess/cloud-computing/the-complete-historyofcloud-computing.jsp .

[3] Armando, F. (2011) "Cloud Computing-What's in it for Me as a Scientist?" Science (331)6016, p. 406.

[4] Barnhill, D.S. (2010) "Cloud Computing and Stored Communications: Another Look at Quon v. Arch Wireless," (Privacy Law) (Annual Review of Law and Technology), Berkeley Technology Law Journal (25), pp. 621-648.

[5] Bret, M. (2009) “In Clouds Shall We Trust?” IEEE Security \& Privacy (7)5, pp. 3-3.

[6] Bhardwaj, S., Jain, L., \& Jain, S. (2010). Cloud Computing: A Study of Infrastructure-as-a-Service .International Journal of Engineering and Information Technology, 60-63

[7] Behrand, T., Wiebe, E. N., London, J. E., \& Johnson, E. C. (2010). Cloud computing adoption and usage in community colleges. Behaviour \& Information Technology, 30(2), 231-240.

[8] Cloud Computing. (2013, January). Retrieved October 5, 2013, from Wikipedia, the free encyclopedia:http://en.wikipedia.org/wiki/Cloud_computing\#History

[9] Choo , K. (2010) .Cloud computing: Challenges and future directions. Trends and Issues in Crime and Criminal

[10] Gupta, A., Pande, P., Qureshi, A. and Sharma, V. (2011). A proposed Solution: Data Availability and Error Correction in Cloud Computing. International Journal of Computer Science and Security, 5(4), 405-413. 
[11] Katzan Jr., H. (2010c) “On the Privacy of Cloud Computing," International Journal of Management and Information Systems (14)2, p. 1

[12] Motta, G., Sfondrini, N. and Sacco, D. (2012), "Cloud Computing: A Business and Economical Perspective," 2012 International Joint Conference on Service Sciences, Ieee, pp. 18-22.

[13] McKendrick, J. (2011). Cloud bursts onto the enterprise mainstream. Database Trends and Applications, December, pp. 2-5.

[14] Ministry of industry and commerce, 2013; TAMKEEN , 2013

[15] Misra, S.C. and Mondal, A. (2010), "Identification of a company's suitability for the adoption of cloud computing and modelling its corresponding return on investment", Mathematical and Computer Modelling, Vol. 53, pp. 504-21

[16] Pandey, S., W. Voorsluys, S. Niu, A. Khandoker, and R. Buyya (2011) "An Autonomic Cloud Environment for Hosting ECG Data Analysis Services," Future Generation Computer Systems(28)1, pp. 147-154, doi:10.1016/j.future.2011.04.022.

[17] R. Buyya, C. S. Yeo, S.Venugopal, J.Broberg, and I. Brandic, "Cloud computing and emerging IT platforms: vision, hype and reality for delivering computing as the 5th utility," Future Gener.Comput. Syst.,vol. 25, pp. 599-616, 2009

[18] Singh, B., 1 October 2011. Cloud Deployment Models - Private, Community, Public, Hybrid with Examples. Techno-Pulse. Available from: http://www.techno-pulse.com/2011/10/cloud-deploymentprivate-public-example.html [Accessed 7 October 2013].

\section{AUTHOR}

Dr Minimol Anil Job is working as an Assistant Professor in ITC Department, in AOU Bahrain Branch. She has more than ten tears of academic experience in the field of computer science and information technology. Her current research interests include Information Technology Management in the specialist area of Database management. She is a certified DBA (MS SQL). She has published several papers in the area of educational technology, e-learning, cloud computing and green computing in international journals. Her further area of interest is software development. She is invloled in many acadmic committees and also working as the Program Coordinator of Information Technology Department in AOU Bahrain Branch. 